\title{
Model Reduction, Centering, and the Karhunen-Loeve Expansion
}

\author{
Sonja Glavaški, Jerrold E. Marsden, and Richard M. Murray ${ }^{1}$ \\ Control and Dynamical Systems, 107-81 \\ California Institute of Technology \\ Pasadena, CA 91125 \\ sonja@cds.caltech.edu, marsden@cds.caltech.edu, murray@indra.caltech.edu
}

\begin{abstract}
We propose a new computationally efficient modeling method that captures a given translation symmetry in a system. To obtain a low order approximate system of ODEs, prior to performing a Karhunen Loeve expansion, we process the available data set using a "centering" procedure. This approach has been shown to be efficient in nonlinear scalar wave equations.
\end{abstract}

\section{Introduction}

The classical approach to model reduction of nonlinear systems (see [1]) using the Galerkin method and the Karhunen-Loeve Expansion (KLE) attempts to find an approximate solution of a PDE in the form of a truncated series expansion given by

$$
\hat{u}(x, t)=\sum_{n=1}^{N} a_{n}(t) \varphi_{n}(x)+\bar{u}(x),
$$

where the mode functions $\varphi_{n}(x)$ are based on empirical data and are generated by the standard KLE methods, and $\bar{u}(x)=\lim _{T \rightarrow \infty} \frac{1}{T} \int_{0}^{T} u(x, t) d t$. For systems with rotational (periodic) symmetry, the mode functions are Fourier modes and the order of the reduced model determined by reasonable criteria for the truncation point need not be small. For a PDE with a traveling wave as a solution, normally this approach will not give satisfactory results.

In this paper, we propose a new computationally efficient modeling method that captures a translation symmetry in a system by finding an approximate solution of the governing PDE in the form of a truncated series expansion given by

$$
\hat{u}(x, t)=\sum_{n=1}^{N} a_{n}(t) \varphi_{n}(x+d(t)) .
$$

To generate an optimal set of basis functions $\varphi_{n}(x)$, prior to performing KLE we process the available data set using a "centering" procedure which involves giving an appropriate definition of the center of a wave and moving it to a standard position. The eigenvalues of the covariance matrix of the "centered" data decay rapidly and we obtain a low order approximate system of ODEs. The method may be viewed as a way of implementing the KLE on the space of solutions of the given PDE modulo a given symmetry group. Viewed this way, the methodology is quite general and therefore should be useful in a variety of problems.

To demonstrate the method's performance we applied it to a PDE modeling a deep stall cell phenomena in jet engine compressor systems. Rotating stall is an instability causing a sudden drop in performance of an engine and feedback control is necessary to prevent it from developing. The most preferred approach to control design is to use low order models that adequately describe the basic dynamics of a system. Thus, the three state nonlinear model of Moore and Greitzer (MG3), a Galerkin truncation onto the first Fourier mode of the full Moore-Greitzer model developed in [4] is widely used. The disadvantage of making a Galerkin projection onto a non-propagating function with fixed spatial shape is that it does not properly describe how the stall cell propagates and evolves in simulations. One usually observes that the stall cell quickly develops a square wave spatial structure. There are some recent results [3] in modeling a deep stall cell phenomena leading to the conclusion that stall cell is indeed a rotating square wave. To capture this behavior with nonpropagating modes of fixed spatial shape, one needs to include many modes. The method of centering captures the dynamics of the system with a family of propagating curves and significantly lowers the order of a model obtained.

\section{The Galerkin Projection}

The Galerkin method is a discretization scheme for PDEs based on the separation of variables approach which attempts to find an approximate solution in the 
form of a truncated series expansion given by

$$
\hat{u}(x, t)=\sum_{n=1}^{N} a_{n}(t) \varphi_{n}(x)+\bar{u}(x),
$$

where the $\varphi_{n}(x)$ are known as trial functions. In this way the original infinite dimensional system is approximated by an $N$ dimensional system. We assume that $u$ belongs to a Hilbert space $L_{2}([0,2 \pi])$ with inner product $\langle f, g\rangle=\int_{0}^{2 \pi} f(x) g^{*}(x) d x$.

Suppose we have a system governed by the PDE

$$
\frac{\partial u}{\partial t}=D(u) \quad u:[0,2 \pi] \times(0, \infty) \rightarrow \mathbb{R}
$$

with appropriate boundary conditions and initial conditions, where $D(\cdot)$ is a nonlinear operator that may involve spatial derivatives and/or integrals. To be sure that the original PDE is satisfied as closely as possible by (2) we choose time dependent coefficients $a_{n}(t)$ in such a way that they minimize, with respect to a suitable norm, the residual error produced by using (2) instead of the exact solution. We obtain a reduced order model, namely the system of $N$ ODEs:

$$
\dot{a}_{i}(t)=\left\langle D\left(\sum_{n=1}^{N} a_{n}(t) \varphi_{n}(x)+\bar{u}(x)\right), \varphi_{i}(x)\right\rangle
$$

where $i=1, \ldots, N$. The initial conditions are determined by a system of $N$ linear equations

$$
a_{i}(0)=\left\langle u(x, 0)-\bar{u}(x), \varphi_{i}(x)\right\rangle
$$

where $i=1, \ldots, N$. It is important to notice that to be able to solve the system of ODEs (3) and (4) one only needs to select the set of trial functions $\left\{\varphi_{n}\right\}$ and the initial conditions of the original system $u(x, 0)$. Any complete set of trial functions will suffice, but we focus on those generated by the KLE.

\section{Karhunen-Loeve Expansion}

The Karhunen-Loeve Expansion (KLE) is a well known procedure for extracting a basis for a modal decomposition from an ensemble of signals, such as data measured in the course of an experiment. Its mathematical properties, especially, optimality suggest that it is the preferred basis to use in many applications. The Karhunen-Loeve expansion provides the most efficient way of capturing the dominant components of an infinite-dimensional process with surprisingly few modes. The KLE technique was introduced in the context of turbulence by Lumley (see [2]) in the late sixties to analyze experimental data aiming to extract typical patterns in space and time.

The fundamental idea behind KLE is very pragmatic. Suppose we have an ensemble $\left\{u^{(k)}\right\}$ of scalar fields, each being a function $u^{(k)}=u^{(k)}(x)$ defined for $x \in$ $[0,2 \pi]$. To find a good representation of members of $\left\{u^{(k)}\right\}$, we will need to project each $u^{(k)}$ onto candidate basis functions, so we assume that the $u$ 's belong to a Hilbert space $L_{2}([0,2 \pi])$.

We want to find a basis $\left\{\varphi_{n}\right\}$ for $L_{2}([0,2 \pi])$ that is optimal for the given data set in the sense that the finite-dimensional representation of the form

$$
\hat{u}(x, t)=\sum_{n=1}^{N} a_{n}(t) \varphi_{n}(x)+\bar{u}(x)
$$

describes typical members of the ensemble better than representations of the same dimension in any other basis. To simplify notation we introduce the variation of $u(x, t)$ from the mean $\bar{u}(x)$ and denote it as $v(x, t)$. The notion of typical implies the use of time averages over an ensemble $\left\{u^{(k)}\right\}$ and optimality is equivalent to maximizing the averaged normalized projection of $v(x, t)$ onto $\left\{\varphi_{n}\right\}$.

In general, the existence of the expansion (5) is guaranteed under certain conditions by the Karhunen-Loeve expansion theorem (see [6]), that also provides us with a method for constructing the orthonormal set of functions $\left\{\varphi_{n}\right\}$ and the uncorrelated set of coefficients $\left\{a_{n}\right\}$.

The orthonormal basis functions $\left\{\varphi_{n}(x)\right\}$ are found via the integral equation

$$
\int_{0}^{2 \pi} R(x, y) \varphi_{n}(y) d y=\lambda_{n} \varphi_{n}(x)
$$

where

$$
R\left(x, x^{\prime}\right) \doteq E\left(v(x) v^{*}\left(x^{\prime}\right)\right),
$$

and $E(\cdot)$ stands for time averaging, i.e.

$$
E(f(\cdot))=\frac{1}{T} \lim _{T \rightarrow \infty} \int_{0}^{T} f(t) d t .
$$

Thus, the optimal basis is given by the eigenfunctions $\left\{\varphi_{n}\right\}$ of the integral equation (6) whose kernel is the averaged autocorrelation function and in the rest of the paper they will be called empirical eigenfunctions. If we define the mean energy projection to be $E\left[\left|\left\langle v, \varphi_{n}\right\rangle\right|^{2}\right]$, then the eigenvalues $\left\{\lambda_{n}\right\}$ corresponding to an empirical eigenfunction may be interpreted as the "the mean energy of the process $v(x, t)$ projected on the $\varphi_{n}$ axis in function space."

Assume that the eigenvalues $\left\{\lambda_{n}\right\}$ corresponding to $\left\{\varphi_{n}\right\}$ have been ordered so that $\lambda_{i+1}>\lambda_{i}$ for all $i$. It can be shown that if $\left\{\psi_{n}\right\}$ is some arbitrary set of orthonormal basis functions in which we expand $v(x, t)$, then for any value of $N$

$$
\sum_{n=1}^{N} E\left[\left|\left\langle\varphi_{n}, v\right\rangle\right|^{2}\right] \geq \sum_{n=1}^{N}\left[\left|\left\langle\psi_{n}, v\right\rangle\right|^{2}\right] .
$$


Therefore, for a given number of modes $N$ the projection on the subspace used for modeling the flow will on average contain the most energy possible compared to all other linear decompositions.

We consider a linearly independent set of snapshot data samples $\left\{u^{(1)}, u^{(2)}, \ldots, u^{(M)}\right\}$ which is either a result of a performed physical experiment or generated as the numerical solution to a scalar nonlinear PDE. The averaged snapshot is computed as $\bar{u}=\frac{1}{M} \sum_{k=1}^{M} u^{(k)}$ and the mean adjusted snapshots are given by $v^{(k)}=$ $u^{(k)}-\bar{u}$.

For computational purposes, we discretize the spatial domain, which usually leads to a very large spatial correlation matrix and determining the corresponding eigenvalue decomposition is extremely costly. Assuming that $u(x, t)$ is an ergodic process, meaning that time averages equal ensemble averages for each fixed value of $x$, we can represent the averaged spatial correlation function as

$$
R(x, y)=\lim _{T \rightarrow \infty} \frac{1}{T} \int_{0}^{T} v(x, t) v(y, t) d t .
$$

The problem of finding empirical eigenfunctions then reduces to finding eigenvectors $f^{n}$ and eigenvalues $\lambda_{n}$ of the $M \times M$ matrix $C$ whose elements are given by

$$
(C)_{i j}=\frac{1}{M} \int_{0}^{2 \pi} v^{(i)}(x) v^{(j)}(x) d x
$$

where $i, j=1, \ldots, M$. The empirical eigenfunctions are then computed as linear combinations of the data snapshots via

$$
\varphi_{n}(x)=\left[v^{(1)}(x) \ldots v^{(M)}(x)\right] f^{n}
$$

where $n=1,2, \ldots, M$. This approach is known as the method of snapshots.

\section{Symmetry and the Karhunen-Loeve Expansion}

Physical systems may exhibit various types of both continuous and discrete symmetries. It is of a great importance to note that while a physical system or its dynamical system model may well admit a symmetry group, one can not expect ensembles of observations to share the full underlying symmetry group. A simple example of this would be a system with several distinct attractors. Then the time average of a single solution will reproduce just one of these attractors and empirical eigenfunctions generated by time averaging data snapshots obtained in one experimental run have less symmetry than the original problem. Let

$$
\dot{u}=f(u)
$$

be an $n$-dimensional system of ODEs and $\Gamma$ be a symmetry group acting on the phase space $\mathbb{R}^{n}$, where the elements $\gamma$ of $\Gamma$ are $n \times n$ matrices. The equation (7) is said to be equivariant under $\Gamma$ if for every $\gamma \in \Gamma$ the equation

$$
\gamma f(u)=f(\gamma u)
$$

holds. This implies that if $u$ is a solution of (7), then so is $\gamma u(t)$.

Adopting the same philosophy for the KLE concept leads to the conclusion that if $\varphi_{n}$ and $\lambda_{n}$ are the empirical eigenvectors and corresponding eigenvalues generated from a set of experiments $\left\{u^{(k)}\right\}$ of a dynamical system equivariant under a group $\Gamma$ then a necessary condition for the system generating $\left\{u^{(k)}\right\}$ to be ergodic is that each of the finite dimensional eigenspaces corresponding to a given empirical eigenvalue be invariant under $\Gamma$. This can easily be checked experimentally. An alternative approach is to assume that a system is ergodic and use its known symmetries to increase the size of the ensemble, generating a symmetric data set $\left\{\gamma u^{(k)}\right\}$ from the available measured ensemble $\left\{u^{(k)}\right\}$. This approach has been advocated by Sirovich in [5].

Because of the nature of the stall cell phenomena, we are interested in rotational symmetry, (called homogeneity in the turbulence literature). In this case the averaged two point correlation $R(x, y)$ is homogeneous meaning that it depends only on the difference of the two coordinates, and the eigenfunctions of the integral equation (6) are Fourier modes. Thus, homogeneity completely determines the form of the empirical eigenfunctions, whereas ordering of the eigenvalues depends on the Fourier spectrum of the data involved.

In the case of a stall cell where we have a square like spatial structure the disadvantage of making a Galerkin truncation of corresponding ensemble of data onto Fourier modes is that to capture how the stall cell propagates and evolves, one needs to include a large number of modes. A natural remedy for this is to try to capture the dynamics with a family of propagating curves. We will concentrate on that approach in the next section.

\section{Centering}

Starting with this section and throughout the paper we will concentrate on systems with rotational symmetry. To illustrate the ideas, we will consider systems governed by the following type of PDE

$$
\frac{\partial u}{\partial t}+\omega \frac{\partial u}{\partial x}=D(u) \quad u:[0,2 \pi] \times(0, \infty) \rightarrow \mathbb{R}
$$

with periodic boundary conditions, $u(0, t)=u(2 \pi, t)$, and where $D(\cdot)$ is a nonlinear operator that may involve 
higher order spatial derivatives. In general these PDEs have a traveling (rotating) wave solution and we would like to obtain as few as possible modes necessary to accurately approximate shape of a propagating wave. To accomplish this, we have to separate the movement of a solution $u(x, t)$ from the evolution of a wave shape.

First, we define a center of each member of an available ensemble $\left\{u^{(k)}\right\}$

Definition 5.1 Let $f(x)$ be a periodic function defined on $[0,2 \pi]$ with period $2 \pi, f(x)=f(x+2 \pi)$. Define the center $C$ of $f(x)$ to be the number $C$ satisfying

$$
\int_{0}^{C}[f(x)]^{2} d x=\int_{C}^{2 \pi}[f(x)]^{2} d x .
$$

In order to extract the propagating wave we position all data snapshots so that their centers are at the same point. For simplicity and without loss of generality, we have chosen to place data snapshots centers at $\pi$ so that, for a suitable constant $d$,

$$
\int_{0}^{\pi}[f(x-d)]^{2} d x=\int_{\pi}^{2 \pi}[f(x-d)]^{2} d x .
$$

We call this procedure the centering of a wave. It is performed using the following iterative procedure.

1. Start with a snapshot $u^{t}(x)=u(x, t)$

2. Compute the center $C$ of the wave $u^{t}(x)$

3. Shift the wave $u^{t}(x)$ to the right by $|\pi-C|$

4. Find the center of $u^{t}(x)$

5. If converged $(C=\pi)$, then stop, else go to 3

Suppose that we have an ensemble $\left\{u^{(k)}\right\}$ of scalar fields, each being a function $u^{(k)}=u^{(k)}(x)$ defined for the $x \in[0,2 \pi]$. To find a good representation of the members of $\left\{u^{(k)}\right\}$, we center each member of an ensemble to obtain a centered data ensemble $\left\{\left(u^{c}\right)^{(k)}\right\}$ and then project each $\left(u^{c}\right)^{(k)}$ onto candidate basis functions. Because we assumed that the $u$ 's belong to a Hilbert space $L_{2}([0,2 \pi])$, this also holds for the $u^{c}$ s.

Performing KLE on the centered data set, we find a basis $\left\{\varphi_{n}\right\}$ for $L_{2}([0,2 \pi])$ that gives a finite-dimensional centered data representation of the form

$$
\hat{u}^{c}(x, t)=\sum_{n=1}^{N} a_{n}(t) \varphi_{n}(x)+\bar{u}^{c}(x) .
$$

The original ensemble is then approximated as

$$
\hat{u}(x, t)=\sum_{n=1}^{N} a_{n}(t) \varphi(x+d(t))+\bar{u}^{c}(x+d(t)) .
$$

\section{The Reduced Order Model}

Suppose we have a system governed by the PDE (8). The original attempt was to find an approximate solution in the form of a truncated series expansion given by

$$
\hat{u}(x, t)=\sum_{n=1}^{N} a_{n}(t) \varphi_{n}(x+d(t))+\bar{u}^{c}(x+d(t)),
$$

where the $\varphi_{n}(x)$ are trial functions obtained after performing KLE on the centered data ensemble $\left\{\left(u^{c}\right)^{(k)}\right\}$. This way the original infinite dimensional system is again approximated by an $N$ dimensional system.

To ensure that the original PDE is satisfied as closely as possible by (9) we choose time dependent coefficients $a_{n}(t)$ so that the residual error produced by using (9) instead of the exact solution is minimized. At any time $t$ we want the residual

$$
r(x, t)=\frac{\partial \hat{u}(x, t)}{\partial t}+\omega \frac{\partial \hat{u}(x, t)}{\partial x}-D(\hat{u}(x, t))
$$

to be orthogonal to a chosen number of trial functions, i.e.,

$$
\left\langle r(x, t), \varphi_{i}(x+d(t))\right\rangle=0, \quad i=1, \ldots, N .
$$

Substituting (9) into (10) yields,

$$
\begin{aligned}
& r(x, t)=\sum_{n=1}^{N} \dot{a}_{n}(t) \varphi_{n}(x+d(t))+ \\
& (\dot{d}+\omega)\left(\sum_{n=1}^{N} a_{n}(t) \varphi_{n}^{\prime}(x+d(t))+\bar{u}^{c^{\prime}}(x+d(t))\right)- \\
& D\left(\sum_{n=1}^{N} a_{n}(t) \varphi_{n}(x+d(t))+\bar{u}^{c^{\prime}}(x+d(t))\right)
\end{aligned}
$$

Applying the orthogonality condition and using the orthonormality property of the set of trial functions results in a reduced order model which is a system of $N$ ordinary differential equations

$$
\begin{aligned}
\dot{a}_{i}(t)= & (\dot{d}+\omega) \sum_{n=1}^{N} a_{n}(t) \beta_{i n}-\left\langle\alpha_{n}, \varphi_{i}(x+d(t))\right\rangle+ \\
& (\dot{d}+\omega)\left\langle\bar{u}^{c^{\prime}}(x+d(t)), \varphi_{i}(x+d(t))\right\rangle
\end{aligned}
$$

where

$$
\begin{gathered}
\beta_{i n}=\left\langle\varphi_{i}(x+d(t)), \varphi_{n}^{\prime}(x+d(t))\right\rangle \\
\alpha_{n}=D\left(\sum_{n=1}^{N} a_{n}(t) \varphi_{n}(x+d(t))+\bar{u}^{c}(x+d(t))\right) .
\end{gathered}
$$

The initial conditions for the resulting system of ODEs are determined by a second application of the Galerkin 
approach. We force the residual of the initial conditions $r_{0}(x)=u(x, 0)-\hat{u}(x, 0)$ to be orthogonal to the first $N$ basis functions and we obtain a system of $N$ linear equations.

$$
a_{i}(0)=\left\langle u(0, x)-\bar{u}^{c}\left(x+d_{0}\right), \varphi_{i}\left(x+d_{0}\right)\right\rangle .
$$

Centering separates the evolution of the wave shape and movement of the wave. The system of ODEs (11) and (12) model the the evolution of the wave shape. We assume that propagation of the wave can be represented by the movement of its center, and a single ODE modeling movement of the wave center can be extracted from $d(t)$ obtained by centering. In the case when $d(t)$ depends linearly on time, and (this holds for the examples we have considered) waves rotate with a constant speed an ODE modeling $d(t)$ is

$$
d(t)=d_{0}+\dot{d}(t) t, \quad \dot{d}(t)=-\omega .
$$

Note that to solve the system of ODEs (11), (12), and (13) one needs to select the set of trial functions $\left\{\varphi_{n}\right\}$, the initial conditions of the original system $u(x, 0)$, and the initial condition for $d(t)$. We choose trial functions generated by the KLE performed on the "centered" data snapshots, and propose determining $d_{0}$ by centering the first data snapshot.

\section{Computational Results}

In this section we will apply model reduction by centering and KLE to the equation modeling the unsteady axial flow in the compression system introduced in [3]. The rotating stall is treated as a large-scale phenomenon, and is a feature of the average flow. The averaging volume is extended over rotor and stator rows and many blades, and the role of velocity fluctuations in deep stall instability is emphasized. The PDE is

$$
\frac{\partial u}{\partial t}+\omega \frac{\partial u}{\partial x}=f(u)-\langle f(u)\rangle+\gamma \frac{\partial^{2} u}{\partial x^{2}},
$$

where $u$ is the axial velocity, $t$ is time, $x$ is angular variable, $\omega$ is the velocity of stall cell rotation, $f(u)$ is the compression system characteristic function, and $\langle f(u)\rangle$ is the annulus average of the characteristic function

$$
\langle f(u)\rangle=\frac{1}{2 \pi} \int_{0}^{2 \pi} f(u) d x .
$$

14 is a non dimensional reaction-diffusion type equation, often with cubic nonlinearity. The steady state, nonuniform solutions of the equation (14) are the stall cells that rotate around the annulus with the average velocity being half the rotor velocity. The numerical existence of such solutions has been shown in [3].

In this section we will show the results of simulation of the equation (14) carried out with $\omega=0.5$ and $\gamma=0.01$. The evolution of a small sinusoidal disturbance superimposed on the uniform flow for the mean flow $\Phi=0.3$ is shown in the Figure 1. Figure 2 shows

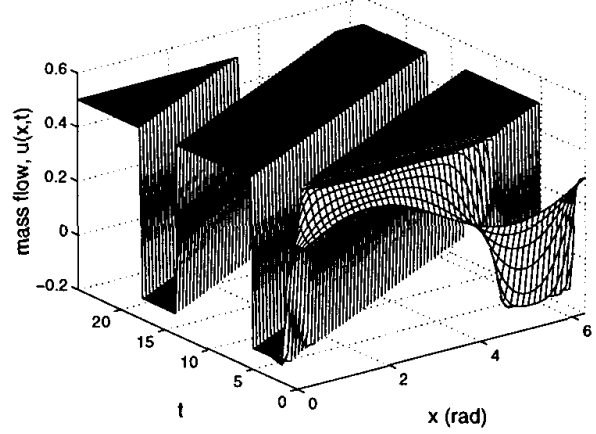

Figure 1: Stall cell evolution, $\Phi=0.3$.

a comparison of the mean snapshot of the original data ensemble and the mean snapshot of the centered data ensemble. The square wave shape appears immediately in the shape of a mean snapshot of the centered data ensemble, whereas the mean snapshot of the original data ensemble gives no helpful information about the shape of the rotating wave. Figure 3 shows a comparison of the first two standard and centered KL modes. The first two centered modes contain more than $96 \%$ of the energy of the data ensemble. The first two KL modes contain a bit more than $67 \%$ of the original data ensemble energy. Because the square wave develops rather quickly, most of the data snapshots are just rotated versions of a square shape, meaning that even though the system does not exhibit strict $S O(2)$ symmetry, the KL modes obtained are just Fourier modes.

Figure 4 shows one of the data snapshots used and its

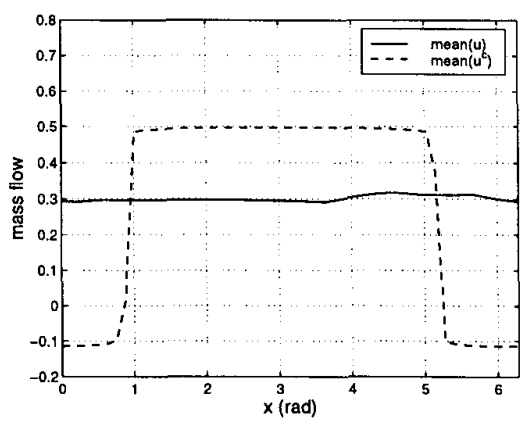

Figure 2: Mean data of original and centered data snapshots

reconstructions $r u(x, t)$ using the first five $\mathrm{KL}$ modes and its reconstructions $r u^{c}(x, t)$ using only the first two centered $\mathrm{KL}$ modes. It is clear that we are outperforming the classical method by the use of centering.

Once we have extracted centered KL modes, and obtained time varying ODEs that model the deviation of 

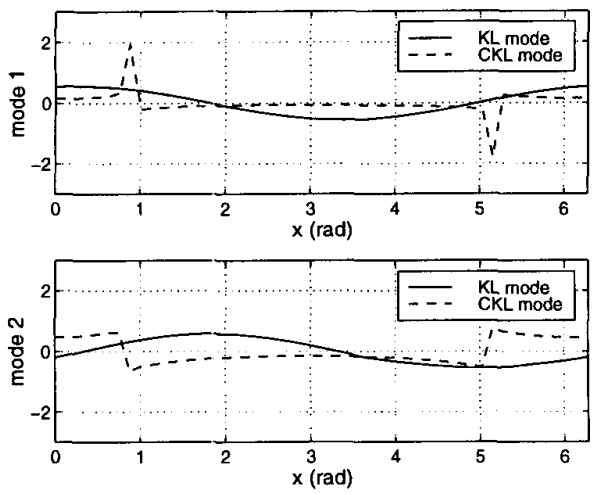

Figure 3: First and second $\mathrm{KL}$ and centered KL mode.

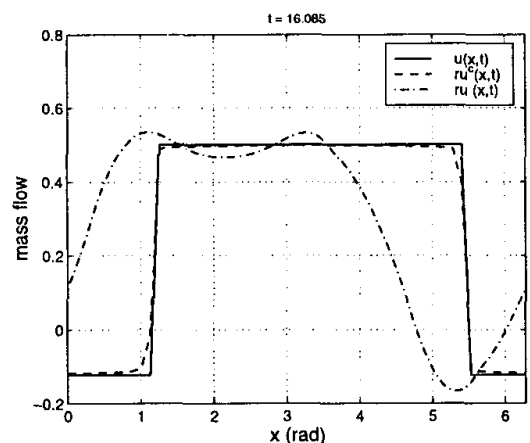

Figure 4: Original snapshot of $u(x, t)$ and its reconstructions by $5 \mathrm{KL}$ and by 2 centered KL modes.

the PDE solution around the mean square wave, we would like to justify our model. Thus, we simulate the original PDE using $u(x, 0)=\sum_{i=1}^{2} a_{i}^{0} \varphi_{i}\left(x+d_{0}\right)$ as an initial condition.

We project the results of the simulation onto previously extracted centered KL modes to obtain a set of ODEs modeling the wave shape evolution, and we simulate them. We also simulate our time varying ODE using $a_{1}^{0}, a_{2}^{0}$, and $d_{0}$ as initial conditions, and denote the computed modal coefficients as $a_{i}^{o}(t)$. In Figure 5 we compare the modal coefficients so obtained. Our reduced order model predicts system behavior rather well.

\section{Conclusions}

In this paper we considered systems governed by PDEs that have a traveling (rotating) wave solution and we have showed how to obtain as few modes as possible to accurately approximate the shape of a propagating wave. We accomplished this by separating the movement of a solution $u(x, t)$ and the evolution of a wave shape. To extract the propagating wave, we position
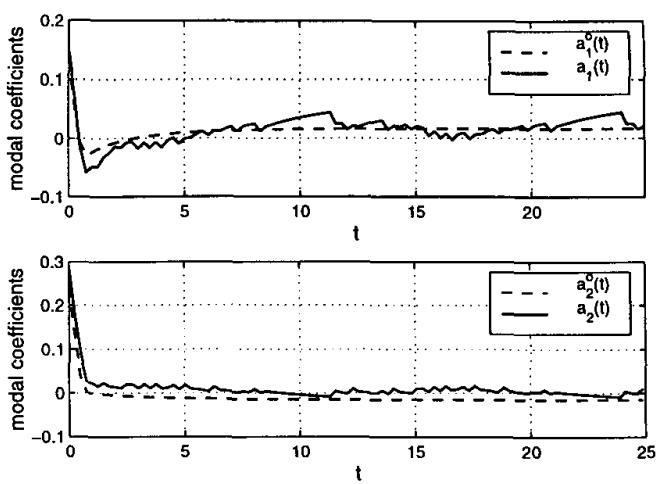

Figure 5: Modal coefficients comparison.

all data snapshots so that their centers lie at the same point. For simplicity and without loss of generality we place data snapshots centers at $\pi$, and we call this procedure the centering of a wave. It is performed using simple iterative procedure.

To demonstrate the method's performance we applied it to a PDE modeling a deep stall cell phenomena (14). It was obvious that we are obtaining more information from the available data by centering, and that we reduce the order of the models needed significantly.

\section{Acknowledgments}

The authors are thankful to Igor Mezić, John Doyle, Michael Dellnitz, and Larry Sirovich for many helpful and educational discussions.

\section{References}

[1] P. Holmes, J. L. Lumley, and G. Berkooz. Turbulence, Coherent Structures, Dynamical Systems and Symmetry. Cambridge University Press, 1996.

[2] J.L. Lumley. Atmospheric Turbulence and Wave Propagation. Nauka, Moscow, 1967.

[3] I. Mezić. A large-scale theory of axial compression system dynamics. In preparation, 1998.

[4] F.K. Moore and E.M.Greitzer. A theory of poststall transients in axial compression systems - Part I: Development of equations. Journal of Eng. for Gas Turbines and Power, 108:68-76, 1986.

[5] L. Sirovich. Turbulence and the dynamics of coherent structures, parts i-iii. Quart. of Appl. Math., XLV:561-582, 1987.

[6] E. Wong. Stochastic Processes in Information and Dynamical Systems. McGraw-Hill, 1971. 